\title{
DESIGN OF A MULTI-CELL, HOM DAMPED SUPERCONDUCTING CAVITY FOR THE STRONG RF FOCUSING EXPERIMENT AT DAФNE
}

\author{
David Alesini, Caterina Biscari, Roberto Boni, Alessandro Gallo, Fabio Marcellini, Mikhail Zobov, \\ INFN/LNF, Frascati, Italy \\ Carlo Pagani, INFN/LASA, Segrate (MI) and DESY, Hamburg
}

\section{Abstract}

A strong RF focusing experiment to be performed at the DAФNE $\Phi$-factory has been proposed to create and observe a bunch length modulation along the ring. The very large RF gradient required to reach the strong focusing regime can only be obtained by using a multicell superconducting cavity. Moreover, in order to demonstrate the feasibility of a high luminosity collider based on this principle, a total multibunch current of the order of $1 \mathrm{~A}$ has to be stored under stable conditions in this regime. A $1.3 \mathrm{GHz} 7$-cells cavity has been designed for this purpose, based on the TESLA geometry with small modifications of the basic cell to comply with the DAФNE revolution frequency. The number of cells has been changed from 9 to 7 to reduce the number of the cavity HOMs, while the beam tubes have been enlarged to let most of the HOMs propagate and be damped by roomtemperature ferrite rings. The modes of the first longitudinal band, which include the accelerating $\mathrm{TM}_{010-\pi}$, do not propagate in the beam tubes and have been studied with special care to prevent the overlap with the bunch revolution harmonics and to cure the effects of coupling to the synchrotron tune sidebands.

\section{INTRODUCTION}

The interest for short and intense bunches is growing in the storage ring physics community. The super-factory projects ask for short bunches to avoid the hourglass effect while reducing the vertical beta-function at the Interaction Point (IP) to increase the luminosity. On the other hand the synchrotron light users are interested in stable production of Coherent Synchrotron Radiation (CSR) that also requires short bunches, which have been so far obtained only at very low current with quasiisochronous lattices.

Table 1: cavity parameters.

\begin{tabular}{|l|c|}
\hline RF frequency [MHz] & 1288.9 \\
\hline Max RF voltage [MV] & 8 \\
\hline R/Q geometric factor $[\Omega]$ & 390 \\
\hline Quality factor (@ 1.8 K) & $10^{10}$ \\
\hline Cavity wall power [W @ 1.8 K] & 8 \\
\hline Loaded quality factor & $2 \div 410^{7}$ \\
\hline $\begin{array}{l}\text { Cavity detuning for Beam Loading } \\
\text { (@ 8 MV, } \mathrm{I}_{\mathrm{b}}=1 \mathrm{~A} \text { ) [kHz] }\end{array}$ & -60 \\
\hline RF generator power [kW] & 1 \\
\hline Cavity length [m] & 0.8 \\
\hline
\end{tabular}

The Strong RF Focusing (SRFF) scheme [1] has been originally proposed to get short and intense bunches at the
IP in the preliminary design of a super $\Phi$-factory aimed at reaching a luminosity of the order of $10^{34} \mathrm{~cm}^{-2} \mathrm{~s}^{-1}$. In such a scheme a large momentum compaction factor together with a very high RF gradient across the bunch provide a modulation of the bunch length along the ring, which can be minimized at the IP.

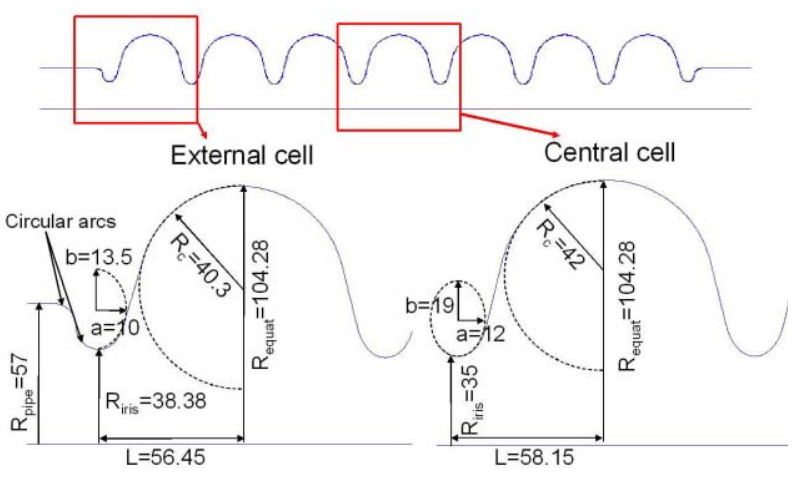

Figure 1: cavity profile and dimensions.

An accelerator physics experiment to be performed at the Frascati $\Phi$-factory DA $\Phi$ NE has been proposed to create and observe for the first time the bunch length modulation along the ring [2]. A $1.3 \mathrm{GHz}$ 7-cells Superconducting (SC) cavity has been designed for this purpose, based on the TESLA [3] geometry. The cavity parameters are shown in Table 1.

\section{CAVITY DESIGN}

The $1.3 \mathrm{GHz} 7$-cells cavity design is based on the TESLA geometry with small modifications of the basic cell to comply with the DAФNE revolution frequency, as shown in Fig. 1. The number of cells has been changed from 9 to 7 to reduce the number of the cavity HOMs, while the beam tubes have been enlarged to let most of the HOMs propagate and be damped by roomtemperature ferrite rings. As an example, the transmission coefficient between the two enlarged beam tubes for the $2^{\text {nd }}$ and $3^{\text {rd }}$ monopole bands is plotted in Fig. 2. The highest quality factors are of the order of $10^{3}$.
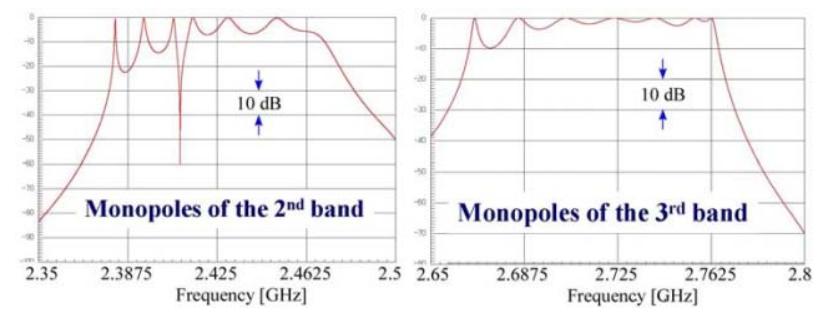

Figure 2: damped monopoles of the $2^{\text {nd }}$ and $3^{\text {rd }}$ band 


\section{Input coupling dimensioning}

The SC cavity will be used as a harmonic cavity in addition to the standard DAФNE NC RF system. The resistive beam loading is therefore negligible in the SC $\mathrm{RF}$ system. The input coupling factor of the SC cavity is defined essentially by the amount of microphonic noise that shakes the SC cavity and continuously displaces its resonant frequency.

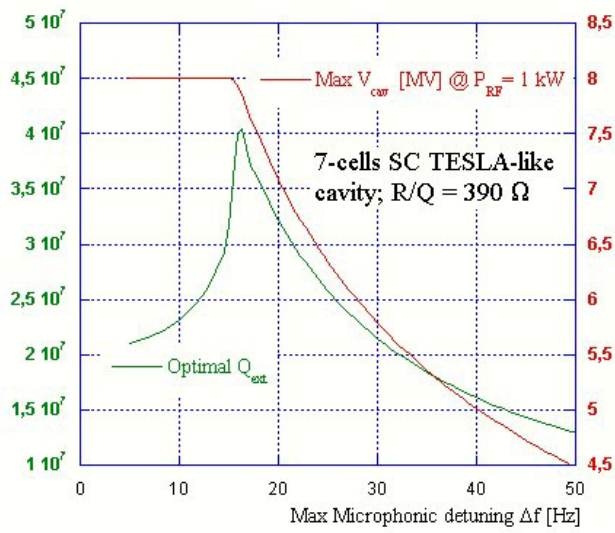

Figure 3: optimal $\mathrm{Q}_{\mathrm{ext}}$ and available accelerating voltage as a function of the cavity microphonic detuning.

The optimum $\mathrm{Q}_{\text {ext }}$ value depends on the maximum tolerable frequency deviation of the accelerating mode, as shown in the plot of Fig. 3. If the microphonic detuning exceeds $\approx 15 \mathrm{~Hz}$, the $1 \mathrm{~kW}$ RF power available from our solid state amplifier is no more sufficient to guarantee $8 \mathrm{MV}$ accelerating voltage. In this case piezo tuners should be used to compensate the microphonic noise, or a feedback loop adapting the RF frequency of the reference oscillator to the instantaneous value of the resonant frequency of the SC cavity accelerating mode has to be implemented. Depending on the amount of the microphonic noise the optimal $Q_{\text {ext }}$ of the input coupler ranges from $2 \cdot 10^{7}$ to $4 \cdot 10^{7}$.
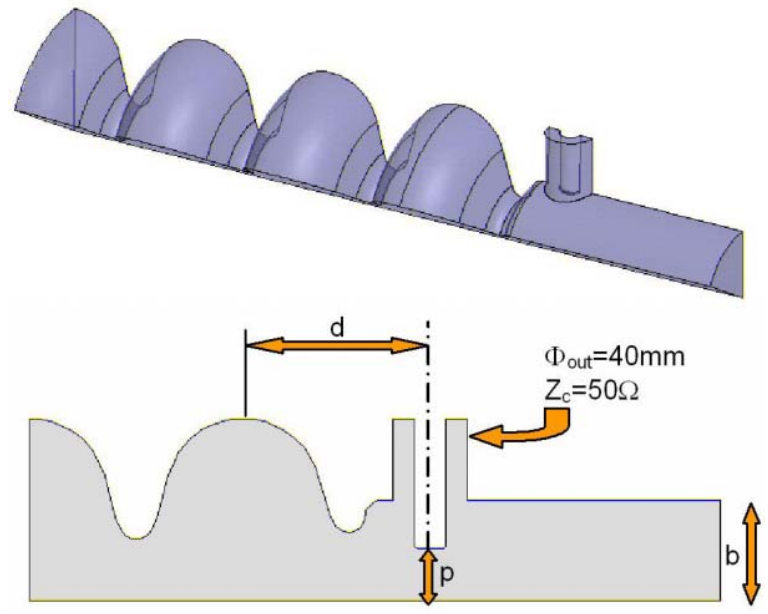

Figure 4: coupler geometry simulated by HFSS.

The input coupler of the TESLA cavity will be used. The coupler position and the inner conductor penetration into the beam tube to get the required input coupling have been defined with 3D simulations using the HFSS code. The geometry shown in Fig. 4 corresponds to $\mathrm{Q}_{\mathrm{ext}} \approx 1 \cdot 10^{7}$ and the quality of the field flatness is excellent as shown in Fig. 5. The TESLA input coupler allows changing the coupling coefficient by varying the penetration of the inner conductor into the beam tube through a bellows system. This feature is very much useful in our case to adapt the coupling to the actual operating conditions.

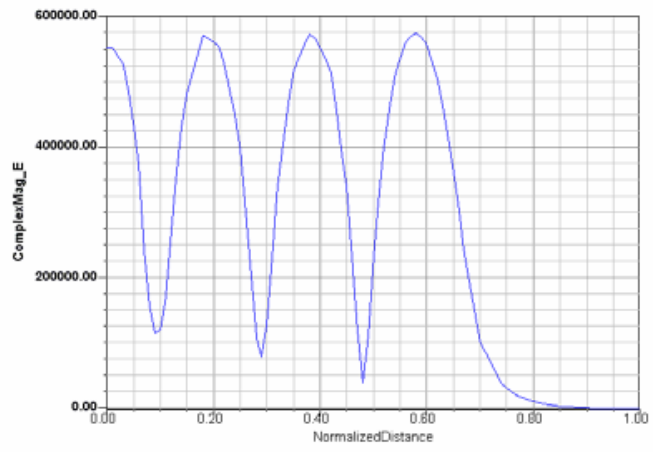

Figure 5: field flatness with coupler as obtained by HFSS.

\section{CONTROL OF THE TM $M_{010}$-LIKE MODES}

The modes of the $1^{\text {st }}$ longitudinal band $\left(\mathrm{TM}_{010}\right.$-like), which include the accelerating one, do not propagate in the beam tubes remaining undamped. To prevent beam disruption caused by the $\mathrm{TM}_{010}$-like mode interaction with the bunch revolution harmonics and/or the synchrotron sidebands a specific strategy based on 2 concepts (mode detuning and direct RF feedback) has been defined.

The first concept is that the resonant frequencies should not overlap the bunch revolution harmonics in order to avoid anomalous power exchange between the beam and the cavity. The nominal resonant frequencies and the R/Q of these modes are reported in Table 2.

Table 2: nominal resonant frequencies an R/Q of the non propagating modes of the $1^{\text {st }}$ monopole band.

\begin{tabular}{|c|c|c|}
\hline Mode & $\boldsymbol{f}[\mathbf{G H z}]$ & $\boldsymbol{R} / \mathbf{Q}[\Omega]$ \\
\hline$\pi / 7$ & 1266.73 & $1 \cdot 10^{-2}$ \\
\hline $2 \pi / 7$ & 1269.90 & $1.4 \cdot 10^{-5}$ \\
\hline $3 \pi / 7$ & 1274.53 & $1 \cdot 10^{-1}$ \\
\hline $4 \pi / 7$ & 1279.72 & $4 \cdot 10^{-4}$ \\
\hline $5 \pi / 7$ & 1284.45 & 1.4 \\
\hline $6 \pi / 7$ & 1287.75 & $1.6 \cdot 10^{-3}$ \\
\hline
\end{tabular}

Due to the cell fabrication tolerances, the real frequency values after fabrication are displaced by few hundreds $\mathrm{kHz}$ respect to the nominal ones and the multi-cell cavity has to be retuned by controlled plastic deformation of the cells to precisely position the frequency of the accelerating mode and restore a good field flatness.

The possibility of tuning the resonant frequency of each mode in the band safely away from the DAФNE bunch 
revolution harmonics during the accelerating mode tuning process has been investigated.

The frequency sensitivity of each mode and the field flatness of the accelerating one have been calculated as a function of the longitudinal deformation of each cell (see Figs. 6 and 7). From these results it is possible to calculate the longitudinal deformation of the cells that we have to implement in the final tuning procedure to shift the resonant frequency of one of the trapped modes by a given quantity. As an example, assuming that we need to shift by $200 \mathrm{kHz}$ a selected mode in the band, we have calculated the set of the cell longitudinal deformations that gives the required shift maximizing the $\mathrm{R} / \mathrm{Q}$ of the accelerating mode and minimizing the frequency shift of the other modes. The results show that, independently of the selected mode, the required cell deformations are $\leq 0.3 \mathrm{~mm}$, while the frequency shift of the other modes is $\leq 200 \mathrm{kHz}$. The accelerating mode R/Q reduction is only few percent.
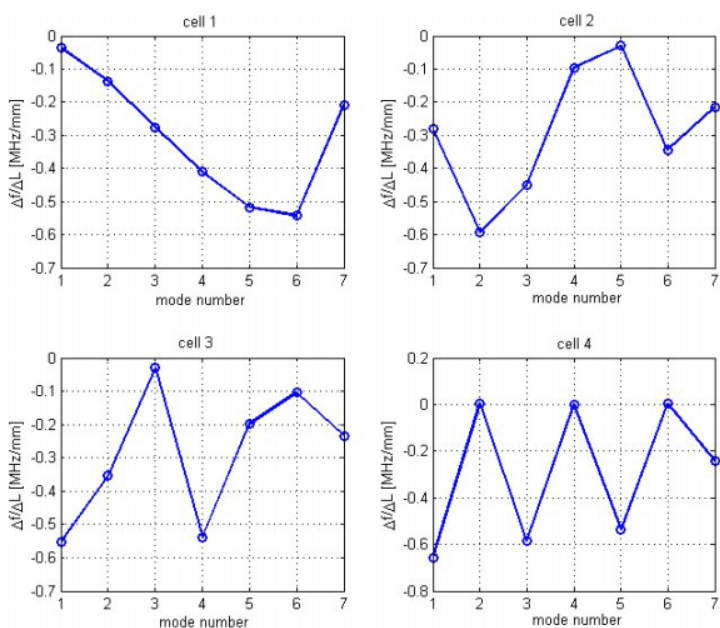

Figure 6: frequency sensitivity of the modes (cell 1 is the first cell).
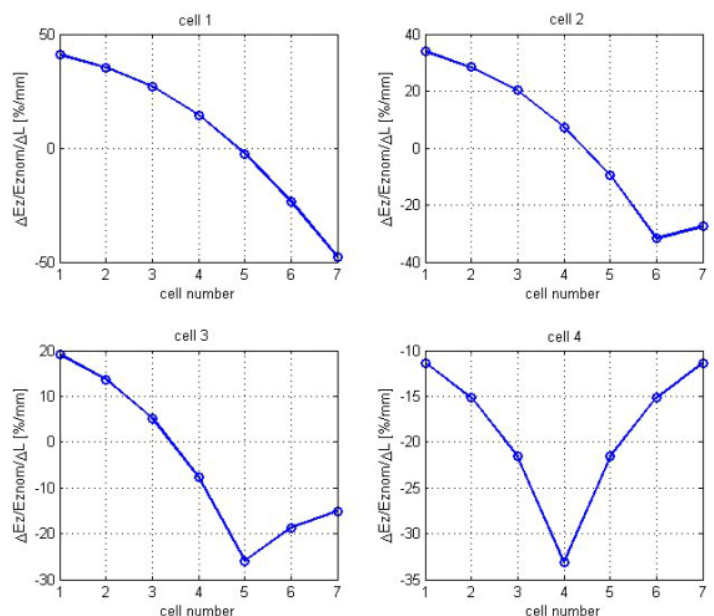

Figure 7: field flatness sensitivity of the modes (cell 1 is the first cell).
The second concept in the $\mathrm{TM}_{010}$-like mode control strategy concerns the cure of the coupled bunch longitudinal instability arising from the coupling with the synchrotron sidebands. To reduce the beam coupling impedance of $1^{\text {st }}$ band monopoles the cavity has to be powered through an efficient direct RF loop as sketched in Fig. 8. The use of a wideband, low group delay solid state amplifier is particularly suitable for this task. A comb filter tuned on the bunch revolution harmonics is necessary to avoid interaction with powerful spectral lines that may saturate the loop and to set optimal phase values of the open loop transfer function at the frequencies where the modes are actually located.

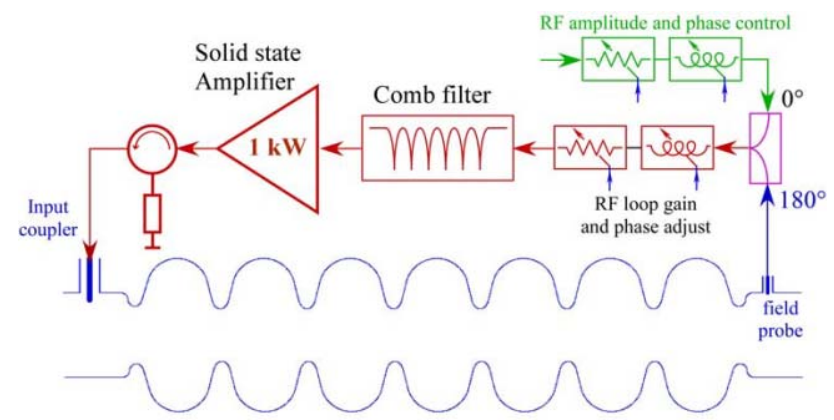

Figure 8: SC cavity powering through a direct RF loop

\section{CONCLUSIONS}

A 7-cells SC cavity based on the TESLA design has been proposed for the bunch length modulation experiment to be made at the Frascati $\Phi$-factory DA $\Phi$ NE. The basic cell has been slightly modified to resonate on the $420^{\text {th }}$ harmonics of the bunch revolution frequency. The high order modes, with the exception of the $\mathrm{TM}_{010^{-}}$ like ones, propagate in enlarged beam tubes and are damped in room-temperature ferrite ring loads. The control strategy of the $1^{\text {st }}$ monopolar band modes $\left(\mathrm{TM}_{010^{-}}\right.$ like), which includes the accelerating one, relies on detuning the modes from the bunch revolution harmonics and impedance reduction through the implementation of a direct RF feedback loop.

The TESLA cavity input coupler allows adjusting the coupling coefficient, whose optimal value is defined by the amount of microphonic noise affecting the cavity accelerating mode.

The cavity design engineering will start after the official approval of the strong RF focusing experiment at DAФNE.

\section{REFERENCES}

[1] A. Gallo, P. Raimondi, M. Zobov, e-Print Archive: physics/0404020.

[2] C. Biscari, "Proposal of an experiment on bunch length modulation in DAФNE", this conference.

[3] B. Aune et al., "Superconducting TESLA cavities", Phys. Rev. ST AB 3, 092001, 2001. 